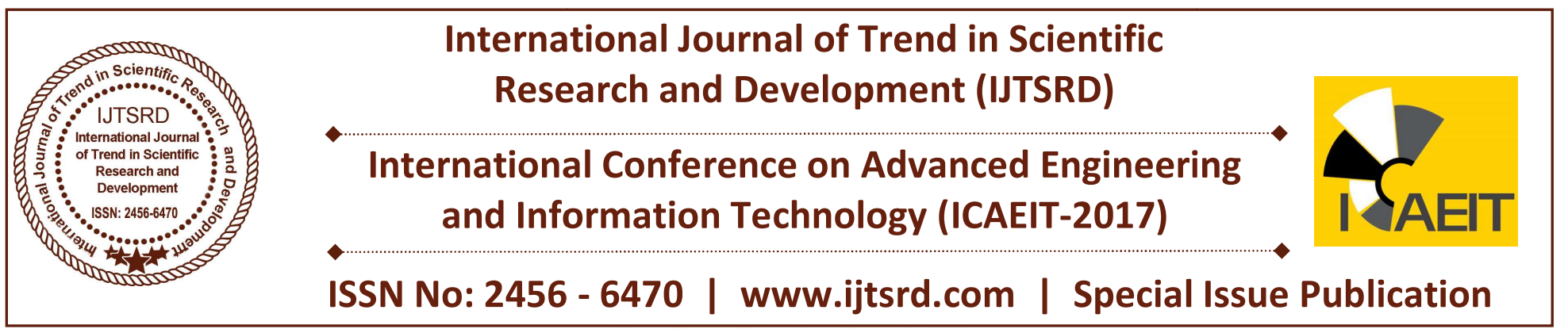

\title{
Coexistence of Zigbee and Wi-Fi Networks in Machine-to-Machine Home System
}

\author{
Wisam Khamil Madhloom ${ }^{1}$, Mia Torres-Dela Cruz ${ }^{2}$, Kayalvizhi Subramanian ${ }^{3}$ \\ ${ }^{1}$ South Russian State Technical University, Novocherkassk, Russia \\ ${ }^{2}$ Faculty of Engineering and Technology, Linton University College, Malaysia \\ ${ }^{3}$ Faculty of Built Engineering, Linton University College, Malaysia
}

\section{ABSTRACT}

The possibility for the home to be networked wirelessly has greatly improved because of emerging technologies. Home networking is very much possible now with the different technologies that are available. This project is an application of two wireless technologies to machine-to-machine (M2M) home networking coexisting together. The networking technologies used and evaluated in the project are ZigBee and Wi-Fi, where the risk of interference in the networks is identified and evaluated.

The M2M home network system that was developed and implemented is an interconnection of electronic home devices grouped by sub-networks categorized by each of the wireless technologies. Because the two sub networks are working in proximity, there is the high chance of interference. Connection for Zigbee include home electronic devices such as light fixtures, television, stereo, etc., and Wi-Fi's network is connections of remotely monitored IP cameras.

The joint application of the two networks showed how these networks affect each other and how they coexist in one system. The paper explored the risk of interference of these two systems when the system is implemented. Guidelines were developed to overcome and mitigate these constraints.

Keywords: Network, Wi-Fi, M2M, Wireless Technologies, ZigBee

\section{INTRODUCTION}

A good home is one that is comfortable, enjoyable, safe, and secure. These are characteristics which are home goals for lots of people. In this era, entertainment, comfort, safety and security are equated to technology and it has hugely helped make homeowners achieve those goals through home networking which has become popular with the proliferation of Internet of Things (IoT). People can now monitor, manage, and secure their homes from a remote location. They are able to operate their home devices and appliances from their jobs or while travelling. They can check what is happening in and around their homes from afar. These are all possible with networking to create a smart home. One method in creating smart home is through Machine to Machine(M2M) home networking [1].

The course of data flow between machine to machine, and eventually machine to human, is what M2M is all about [1]. The usual flow of information is from a machine towards a gateway, through network, which is where a system processes and analyzes it, for whatever time of data or machine there is. However, in M2M, transmission and reception of data remotely is allowed for a machine. M2M is an ideal technology to use in home networking because of its advantages, such as, cost reduction as well as time and effort reduction. As Yue, Jawen, and Rong [2] have stated, M2M networks are characterized by low cost, power, and human intervention. Because of this, the potential is a system that is cost effective and high service quality. 
As home furniture and electronic devices have become more intelligent, the home has improved in terms of comfort, entertainment, security, maintenance, and management. And because of improved communications and networking technologies, connecting these intelligent devices have become a lot easier and gave way to more efficient home networking. Furthermore, the cumbersomeness of cables is being replaced by wireless systems, making networking more manageable than ever.

The project has designed a system where two wireless communication technologies are joined to complement each other in a M2M home network, implemented and evaluated as to their performance regarding functionality and usability. Constraints such as overlap, interference, or signal issues are explored and guidelines were developed to overcome these identified constraints. The two wireless technologies are: ZigBee and Wi-Fi.

\section{Comparing Zigbee and $\mathrm{Wi}-\mathrm{Fi}$}

ZigBee carries the IEEE standard number 802.15.4. This means it belongs to the group that is engaged in network operations and technologies, such as midsized and local networks and is wireless. Baker [3] stated that the home automation standard of ZigBee is an open wireless protocol for personal-area networking (PAN) which was developed in the early 2000 's as a low-cost, low-power alternative to currently available networking protocols. Like ZWave, ZigBee is a "mesh networking" technology, in which packet signals are relayed by adjacent devices within the mesh until they reach their intended target. ZigBee is designed to be very tolerant to radio interference, making it a very good standard for technology-rich smart homes. Therefore, at any time and status, it is appropriate for use and development of intelligent homes [4].

The popular name for wireless local area networks which is based on the IEEE $802.11 \mathrm{~b}$ standard is Wi-Fi [5]. This technology is considered one of the most brilliant areas of the information and communications business [6]. Wi- Fi has taken broad-based interest all over technical and popular areas. It is progressively being viewed as the medium that will bring in several types of technologies that would support or supported by Internet access to the general population [7].
Most Wi-Fi devices in the home network are power intensive. Although the network itself is wireless, the devices are connected to outlets and use a lot of power. For operating networks that are full-scale, Wi$\mathrm{Fi}$ is considered a better choice because faster connection is enabled, base station range is better, and, if configured correctly, has better security.

According to Pothuganti and Chitneni [8], obviously, Wi-Fi provides a higher data rate, compared to Zigbee. Zigbee is well suited for WPAN communication (about $10 \mathrm{~m}$ ), while Wi-Fi is oriented to WLAN (about 100m). When the two wireless technologies were compared and evaluated, tables 1 and 2 below showed the performance of each of the technologies using the criteria specified. The following were measured during testing of the system [9]:

Table 1: Comparative functionalities of Wi-Fi and

\begin{tabular}{|l|l|l|}
\hline \multicolumn{1}{|c|}{ Criteria } & \multicolumn{1}{|c|}{$\begin{array}{c}\text { Zigbee } \\
(802.15 .4)\end{array}$} & $\begin{array}{c}\text { Wi-Fi } \\
(802.11)\end{array}$ \\
\hline $\begin{array}{l}\text { Application } \\
\text { Focus }\end{array}$ & $\begin{array}{l}\text { Electricity switching } \\
\text { control, i.e.lightbulb, } \\
\text { representation of } \\
\text { home electronic } \\
\text { devices }\end{array}$ & $\begin{array}{l}\text { Home } \\
\text { monitoring, } \\
\text { Video and } \\
\text { Photo by } \\
\text { remote } \\
\text { control }\end{array}$ \\
\hline $\begin{array}{l}\text { Transmission } \\
\text { Range(meters) }\end{array}$ & 30 & $1-100$ \\
\hline $\begin{array}{l}\text { Success } \\
\text { Matrix }\end{array}$ & Power, reliability & $\begin{array}{l}\text { Speed, } \\
\text { Flexibility, } \\
\text { Portability }\end{array}$ \\
\hline $\begin{array}{l}\text { Data } \\
\text { Protection }\end{array}$ & 16 bit CRC & 32 bit CRC \\
\hline Optimized for & $\begin{array}{l}\text { Low cost, low } \\
\text { power }\end{array}$ & $\begin{array}{l}\text { Speed, } \\
\text { security }\end{array}$ \\
\hline Frequency & $\begin{array}{l}900 \mathrm{MHz} \text { to 2.4 } \\
\text { GHz }\end{array}$ & $\begin{array}{l}\text { 2.4 and 5 } \\
\text { GHz }\end{array}$ \\
\hline
\end{tabular}

Table 2: Comparative usabilities of Wi-Fi and ZigBee

\begin{tabular}{|l|l|l|}
\hline \multicolumn{1}{|c|}{ Criteria } & \multicolumn{1}{|c|}{$\begin{array}{c}\text { Zig Bee } \\
(802.15 .4)\end{array}$} & \multicolumn{1}{|c|}{$\begin{array}{c}\text { Wi-Fi } \\
(802.11)\end{array}$} \\
\hline $\begin{array}{l}\text { Power } \\
\text { Consumption(days) }\end{array}$ & Very low & High \\
\hline System Resource & $4-32 \mathrm{~KB}$ & $1 \mathrm{MB}$ \\
\hline Battery life(Days) & $100-1000+$ & 5 \\
\hline Bandwidth & $\begin{array}{l}20- \\
250 \mathrm{~KB} / \mathrm{S}\end{array}$ & $11000 \mathrm{~KB} / \mathrm{S}$ \\
\hline Data Rate & $\begin{array}{l}115.2 \\
\text { Kbits/s }\end{array}$ & $\begin{array}{l}11 \text { and } \\
54 \mathrm{Mbits} / \mathrm{s}\end{array}$ \\
\hline Reliability & High & Medium \\
\hline
\end{tabular}


Wi-Fi and ZigBee networks share the same frequency band which is $2.4 \mathrm{GHz}$. Because of this, the two technologies usually operate in proximity and they have to co-exist together. However, WiFi consumes higher power level, while ZigBee uses low power [10].

\section{The Coexistence of the network technologies in M2M home networking}

The Wi-Fi standard operates in two frequency ranges and one of these is the $2.4 \mathrm{GHz}$ ISM (Industrial Scientific and Medical) range which it shares with ZigBee. It is a vulnerable RF spectrum because it is widely used. In a home scenario, it is commonly affected by many other different wireless interferences such as Bluetooth, microwave ovens, baby monitors, surveillance cameras, and wireless telephones [13].

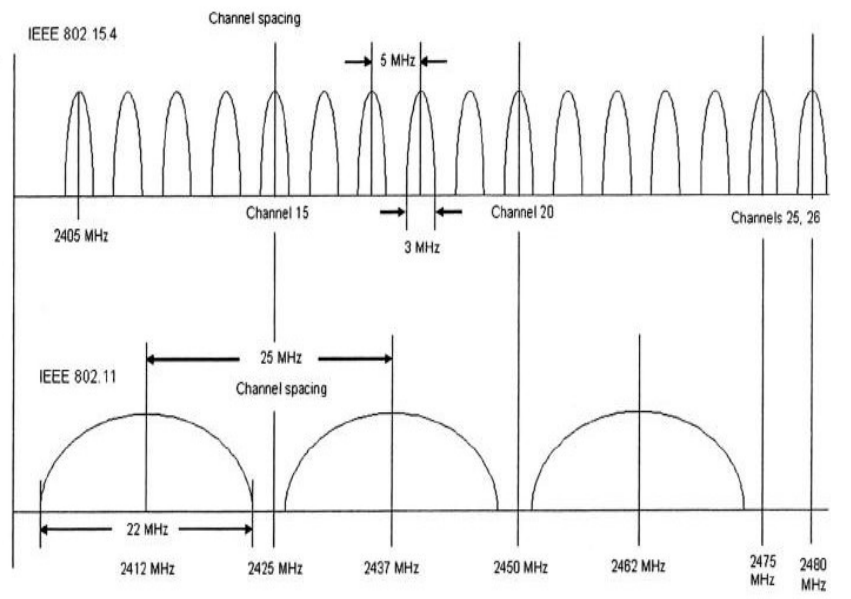

Figure 1: ZigBee (802.15.4) and Wi-Fi (802.11) Channels (Source: Mobiusconsulting.com)

Arif and Supankat [11] stated that interference will occur on networks due to the large number of devices and several different wireless communication technologies are connected. Because of this interference, a decrease in the performance of the wireless networks is noted. Wi-Fi and ZigBee are expected to run simultaneously in close proximity due to the demand for ubiquitous Internet access and increasingly, these two networks are being utilized in urban areas to support real-time and long-term monitoring [10]. Interests in using these two networks to coexist in different systems have intensified and one of these systems is in smart homes. However, because of the overlap in the channels of ZigBee and $\mathrm{Wi}-\mathrm{Fi}$, there is a cause for concern.

Yan, et al [14] stated that ZigBee is vulnerable to WiFi for two reasons, one, the transmission power of
Wi-Fi is approximately 10 to 100 times higher than ZigBee which means it is probably affected by the high power of the Wi-Fi devices; and two, because ZigBee has low data rate and cost, it is inferred that the ability for time resolution becomes slow and unstable. In this research by Yan et. al., they developed a single-antenna sink based design which coexisted with ZigBee and Wi-Fi, called Wizbee. This was developed to apply interference cancelation technique in order to mitigate interference from Wife, and to extract signals from ZigBee.

A series of experiments by Zhao et al [15] resulted in the findings concerning symmetric and asymmetric scenarios in Zigbee-Wi-Fi interference. Accordingly, in symmetric scenarios, ZigBee's throughput degrades heavier than that of $\mathrm{Wi}-\mathrm{Fi}$ and the packet losses is mostly because of ACF. While in asymmetric scenarios, there's a difficulty for ZigBee (802.15.4) to persist because of the interference of Wi-Fi (802.11n), since its Packet Delivery Ratio (PDR) is approaching zero. The reason for the packet losses are because of auto-correlation function (ACF) and corruption.

A simulation by Arif and Supangkat [11] was more related to the system developed in this study because of the similarities of connections and usage of the topologies. Their simulation was done by comparing the throughput of the system by measuring the wireless performance in two ways, one, with only ZigBee and two, with both ZigBee and Wi-Fi simultaneously, in order to find the impact of interference. On the first scenario (ZigBee only), throughput is above $35 \mathrm{Kbps}$. But in the second scenario when Wi-Fi was introduced, the throughput of ZigBee significantly dropped below $35 \mathrm{Kbps}$. From this, the authors inferred that the introduction of WiFi working simultaneously with ZigBee degraded transmission performance of the latter. Extensive experiments were done by Li et al [16] to demonstrate their proposal in tackling issues of the co- existence of ZigBee and $\mathrm{Wi}-\mathrm{Fi}$, which are to shift the paradigm from traditional discrete channel allocation to continuous frequency allocation; innovate in transforming frequency allocation into spatial tessellation in a frequency-distance space, and propose an algorithm to compute a near optimal solution in a localized manner. The authors designed a probabilistic mechanism to adaptively use CSMA according to real- time interference assessments; it can fine-tune the tradeoffs between transmission throughput and quality. 


\section{The System Design}

A M2M home network system was designed, developed and implemented in this project. It is composed of two wireless technology sub networks, Zigbee and Wi-Fi. These two sub networks were designed so that they can be interconnected with electronic home devices and would enable these devices to communicate with each other [1]. Interfaces were developed, which include a central PC interface and mobile interfaces for each sub network, as bridges to integrate the two sub networks so that these can be monitored, either within the home itself or remotely from outside the home. The software for the PC interface and interconnectivity were implemented using $\mathrm{CH}$ and Visual Basic languages. The resulting system is a working home network using the two wireless technologies connected as sub networks, with the devices monitored and optionally controlled from the PC interface and mobile interfaces, with lesser human intervention.

A sample pseudo code below is shown for the algorithm of the system process [1]. The algorithm shows how to perform the different tasks in the system:

\section{$B E G I N$ \\ SELECT TECHNOLOGY (OPTIONS) \\ OPTION 1:// ZigBee Subnetwork \\ SSELECT DEVICE; \\ CONNECT DEVTCE: \\ OPERATE; \\ BREAK, GO TO ALT I; \\ OPTION 2: // Wi-Fi Subnenvork \\ (SELECT TASKS (Settings) \\ SSet Snapshot Directory: \\ Add Camera Porameters: \\ Edit Comera Parameters. \\ Renove Camera Parameters:y}

ZigBee sub network controls devices within the home with shorter range (1-30 meters); those with functionality where electricity switching is involved. While Wi-Fi sub network controls home monitoring in the form of video and photo by remote control.

Figure 1 shows the ZigBee sub network and its components:

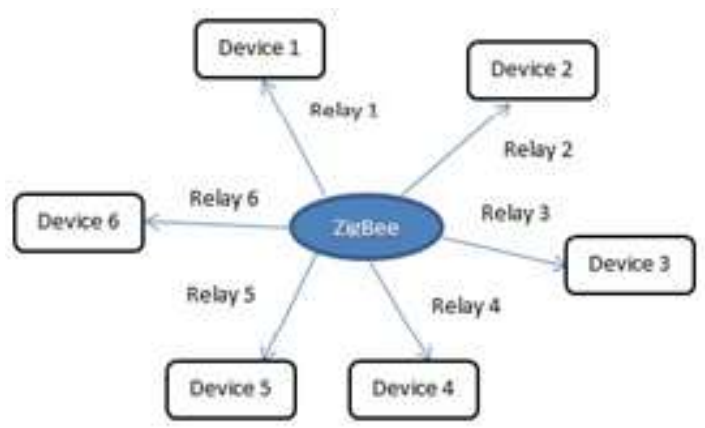

Figure 2: ZigBee Sub network

For Wi-Fi sub network, the objective of the project is to connect IP cameras that can be viewed remotely.

The next figure, Figure 3, is the set-up for Wi-Fi sub network:

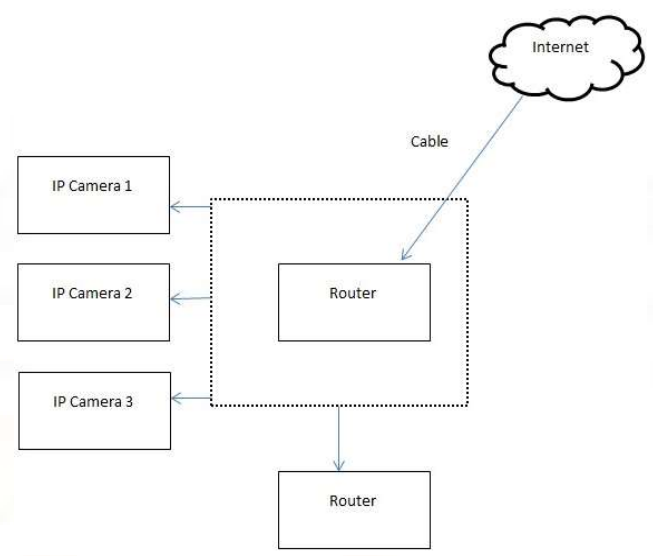

Figure 3: Wi-Fi Sub network

The full system is a combination of sub networks shown in Figure 4:

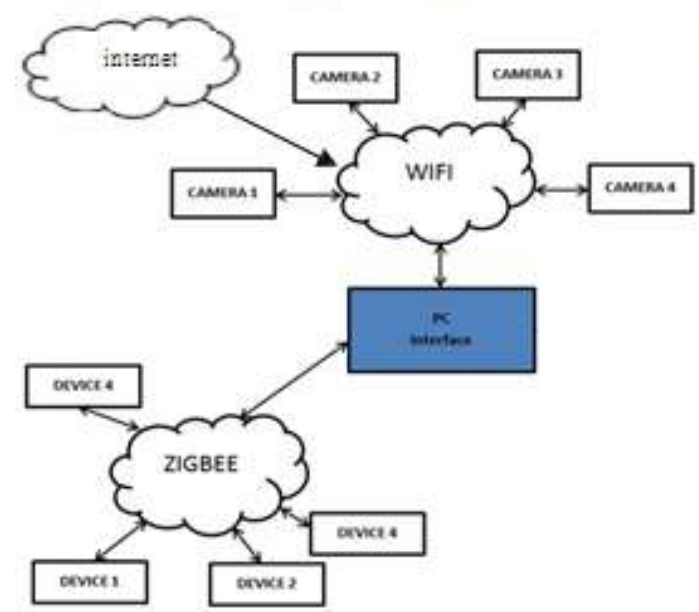

Figure 4: Working System Network

As observed in the set up for the system, issues were detected when the sub networks were run simultaneously. Three set ups were done to observe the signals of the system. These were: Wi-Fi alone, ZigBee alone, and Wi- Fi and ZigBee together. 
a. Wi-Fi alone. This is the normal situation where the performance of the system with only Wi-Fi running is noted. In this set-up, all three IP cameras were launched, initially one at a time and then two, then all three at the same time. Performance of the Wi-Fi was noted and inferred as normal.

b. ZigBee alone. This situation also is the basis for the comparison. ZigBee sub network was launched alone and three representative devices were launched, initially one at a time, then two, then finally all three together.

c. Wi-Fi and ZigBee together. The two networks would be tested for its effect with each other. Both will be deployed simultaneously and will be observed for any difference as compared with running alone.

The result of the evaluation in the Wi-Fi and ZigBee is a positive interference for ZigBee throughput. The signal deteriorated and at some point, the relay for one device did not work. However, Wi-Fi signal has very little change when run simultaneous with ZigBee, with value of change as negligible, as compared with signal when running alone. This corroborates the contention of Arif and Supangkat [11] who did a simulation for ZigBee and Wi-Fi coexistence and measured the throughput for ZigBee alone and then ZigBee together with Wi-Fi. For ZigBee alone, the throughput reached more than $35 \mathrm{Kbps}$ but deteriorated to above $30 \mathrm{Kbps}$ when launched with Wi-Fi (Figure 5).

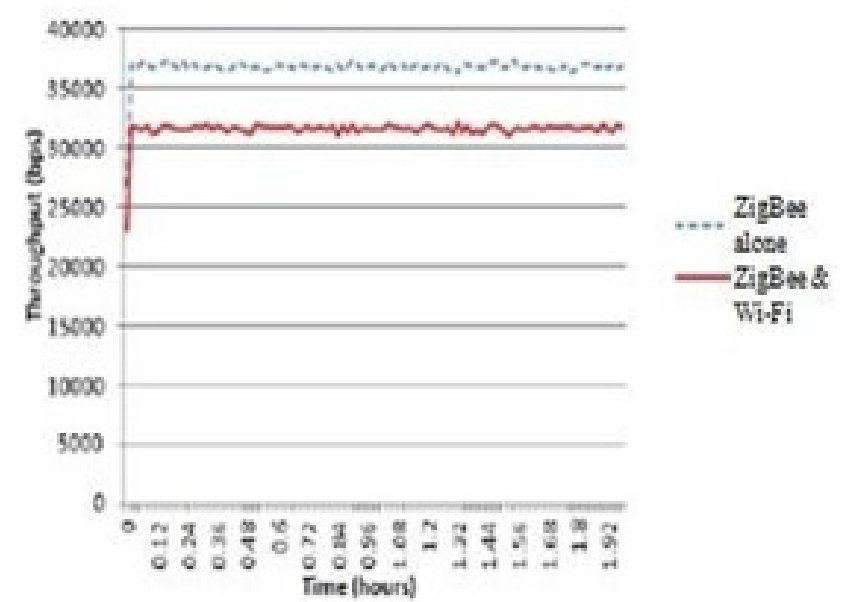

Figure 5: Throughput of ZigBee Alone and ZigBee and Wi-Fi Together (Source: [11])

\section{Solutions for avoiding interference between two networks in M2M Home System}

One solution to avoid interference is to replace Zigbee with a similar low power network such as WizBee.
WizBee [14] is designed to be compatible with current ZigBee and WiFi system, and do not need any protocol modification. The requirement is to replace conventional ZigBee sink with WizBee sink.

On situations where there is interference, Multi Header (MH) solution may be applied. It is effective when there is a loss of ZigBee packet, which is because of header error. Header error is a result of Wi-Fi interference with ZigBee. MH scheme [17] is used to reduce the effective throughput which determines the number of headers adaptively based on the presence of heterogeneous networks. This solution is only effective when interference is present, which allows better throughput and efficiency.

\section{Conclusion}

Coexistence of ZigBee and Wi-Fi in an M2M home networking does makes sense as it would provide convenience of connection and communication within the system. However, there are issues with their coexistence because of their different characteristics.

The project resulted to a well-functioning M2M home network composed of joint wireless technologies, Zigbee and Wi-Fi networks. The coexisting technologies were observed, compared, and documented according to their functionalities and usabilities (tables 1 and 2) in the specific system itself.

The issues of interference between the two network technologies were observed during the test run of the system. It was perceived that when both are deployed at the same time, ZigBee signal deteriorates which makes some devices lose relay from the network. However, when working alone, the signal for ZigBee is of normal rate. Wi-Fi does not seem to get affected by ZigBee at all.

Despite the interference, it is believed that ZigBee and Wi-Fi can coexist together when it can be mitigated through good set-up planning. Solutions are available to avoid or mitigate issues of interference because of ZigBee and Wi-Fi coexistence.

For future work, it is recommended that new systems involving co-existence with ZigBee and Wi-Fi should plan set-up very well to consider new standards which will favor different channels and frequencies to avoid clashing. 
Further studies for other network interference should be done such as ZigBee interference with Bluetooth, ZWave, and other technologies such as Li-Fi is recommended.

\section{References}

1. W. Madhloom and M. Dela Cruz (2015), "M2M Home Network Using 3 Selected Wireless Technologies", http://www.ijetae.com/files/ICSMET15/IJETAE_I CSMET_2015_03.pdf

2. L. Yue, K. Jiawen, and Y. Rong, (2013), "Efficient and Secure Resource Management in Home M2M Networks", International Journal of Distributed Sensor Networks, October 2013.

3. D. Baker, August 16, 2013, How ZigBee Compares in Wireless Home Automation. https://www.control4.com/blog/2013/08/howzigbee- compares-in-wireless-home-automation. [Accessed: 15- Sept- 2017].

4. F. Anan, X. Xiaoling, Y. Wenling, Z. Li, "The Realization of Intelligent Home by ZigBee Wireless Network Technology," Circuits, Communications and Systems, 2009. PACCS '09. Pacific-Asia Conference on , vol., no., pp.81,84, 16-17 May 2009.

5. C. Cordeiro, D. Akhmetov, and M. Park, (2010). IEEE 802.11 ad: introduction and performance evaluation of the first multi-gbps Wi-Fi technology. pp.3--8.

6. "Connect Your Life | Wi-Fi Alliance", Wi-fi.org, 2017. [Online]. Available: https://www.wifi.org/discover-wi-fi/connect-your-life. [Accessed: 15- Sept- 2017].

7. B. Mitchell, "How wireless and computer networks help you do work and have more fun", Lifewire, 07 June 2017. [Online]. Available: https://www.lifewire.com/benefits-of-wirelesshome-computer-networking-816541. [Accessed: 06- Sept- 2017].

8. K. Pothuganti, and A. Chitneni, "A comparative study of wireless protocols: Bluetooth, UWB, Zigbee, and Wi-Fi", Advance in Electronic and Electric Engineering, ISSN 2231-1297, vol. 4, no. 6 (2014), pp. 655-662.
9. W. Madhloom, and M. Dela Cruz, "Comparative M2M Home Network Application of Bluetooth, Zigbee, and Wi-Fi", IACITE 2015, January 2015.

10. S. Jacob, P. Ravi, "Enabling Coexistence of ZigBee and WiFi", Communications on Applied Electronics (CAE) - ISSN : 2394-4714, New York, USA,Volume 2 - No.6, August 2015 www.caeaccess.org

11. S. Arif and S. Supangkat, "Simulation and Analysis of ZigBee - WiFi Interference", ICT For Smart Society (ICISS), 2014 International Conference, IEEE Explore, 24-25 Sept. 2014.

12. Crossbow, 2017. "Avoiding RF Interference Between WiFi and Zigbee" [Online]. Available: https://www.mobiusconsulting.com/ papers/ZigBeeandWiFiInterference.pdf. [Accessed: 08- Oct- 2017].

13. "Wi-Fi RF Spectrum; interferences; how to detect them, case study: microwaves. | Acrylic WiFi", Acrylic WiFi, 2017. [Online]. Available: https:/www.acrylicwifi.com/en/blog/wifi-rfspectrum-detect-study-case-microwave/.

[Accessed: 08- Oct- 2017].

14. Y. Yan, P. Yang, X.Y. Li, Y. Zhang, J. Lu, L. You, J. Wang, J. Han, Y. Xiong. (2015). "WizBee: Wise ZigBee Coexistence via Interference Cancellation with Single Antenna," in IEEE Transactions on Mobile Computing, vol. 14, no. 12, pp. 2590-2603, Dec. 1 2015. doi: 10.1109/TMC.2014.2359673

15. Z. Zhao, X. Wu, X. Lai, J. Zhao, X. Li, "ZigBee vs WiFi: Understanding issues and measuring performances of their coexistence", Performance Computing and Communications Conference (IPCCC), 2014 IEEE International, 5-7 Dec. 2014, Austin, TX, USA.

16. F. Li, J. Luo, G. Shi, Y. He, "ART: Adaptive fRequency-Temporal Co-Existing of ZigBee and WiFi”, IEEE Transactions on Mobile Computing, Vol. 16, No. 3, March 2017.

17. P. D. Sai Manoj, G. V. K. Sasirekha, and J. Bapat, "A Novel Approach for Coexistence of ZigBee with WiFi," International Journal of Computer and Communication Engineering vol. 1, no. 3, pp. 251-269, 2012. 